\title{
Neurological update: treatment escalation in multiple sclerosis patients refractory to fingolimod-potentials and risks of subsequent highly active agents
}

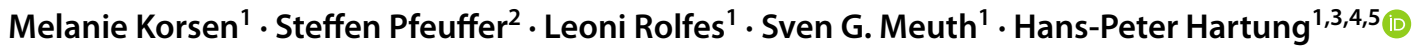

Received: 7 September 2021 / Accepted: 30 December 2021 / Published online: 9 January 2022

(c) The Author(s) 2022

\begin{abstract}
A critical issue in the management of relapsing MS (RMS) is the discontinuation of disease-modifying treatments (DMT) due to lack of efficacy, intolerability or impending risks. With new therapeutic agents introduced into the treatment of RMS, immediate- and long-term consequences of sequential drug use, as well as the effect of the sequence in which the drugs are given, are unclear but may affect efficacy, adverse events, and long-term immunocompetence. In the absence of clinical studies specifically addressing these concerns, observations from clinical practice are of particular value in guiding current management algorithms. Prompted by a study published by Ferraro et al. in this journal, we set out to provide an overview of the published real-world evidence on the effectiveness and safety of switching from fingolimod to another DMT in patients with active RMS. Seventeen publications reporting relevant information were identified. The literature suggests that immune cell depletion induced by alemtuzumab or ocrelizumab is associated with an increased risk of relapse and worsening disability in patients switching from fingolimod compared to patients switching from other therapeutic agents. However, the evidence reported for natalizumab and cladribine is inconclusive. While shortening of the washout period may limit early disease reactivation after fingolimod discontinuation, there is no strong evidence that the duration of the washout period or the absolute lymphocyte count at baseline are predictors of attenuated long-term efficacy. Further real-world studies are required to better understand outcomes among patients who are under-represented in controlled trials.
\end{abstract}

Keywords Adverse events $\cdot$ Disease-modifying treatment $\cdot$ Effectiveness $\cdot$ Fingolimod $\cdot$ Multiple sclerosis $\cdot$ Switch

\section{Introduction}

The expansion of the treatment landscape in active relapsing multiple sclerosis (RMS) has led to increased complexity of treatment choices. Treatment options currently compromise immune cell sequestering substances, such as

Melanie Korsen and Steffen Pfeuffer contributed equally.

Hans-Peter Hartung

hans-peter.hartung@uni-duesseldorf.de

Melanie Korsen

melanie.korsen@med.uni-duesseldorf.de

Steffen Pfeuffer

steffen.pfeuffer@1wl.org

Leoni Rolfes

leoni.rolfes@med.uni-duesseldorf.de

Sven G. Meuth

sven.meuth@uni-duesseldorf.de the sphingosine-1-phosphate receptor blocker fingolimod and the monoclonal antibody natalizumab, as well as celldepleting agents, such as the small molecule drug cladribine and the monoclonal antibodies alemtuzumab (anti-CD52), ocrelizumab (anti-CD20) and ofatumumab (anti-CD20) [1]. They can be administered as a first-line treatment, but most patients are initially treated with low-risk platform agents and then switched to these drugs due to insufficient disease

1 Department of Neurology, Medical Faculty, Heinrich-HeineUniversity Düsseldorf, Moorenstraße 5, 40225 Düsseldorf, Germany

2 LWL-Clinics Muenster, Muenster, Germany

3 Brain and Mind Centre, University of Sydney, Sydney, Australia

4 Department of Neurology, Medical University of Vienna, Vienna, Austria

5 Department of Neurology, Palacky University Olomouc, Olomouc, Czech Republic 
control (so-called 'treatment escalation'). While several studies have examined this treatment strategy $[2,3]-$ the progression from first- to second-line therapy-evidence regarding a 'lateral switch' to a drug with comparable efficacy but different mode of action, following previous active treatment, remains scarce. This is mainly due to phase III clinical trials supporting the approval of these disease-modifying therapies (DMT) not usually including patients on prior active treatment. Consequently, considerations regarding efficacy as well as a comprehensive risk-benefit assessment critically depend on the evaluation of data collected by comparative observational studies done in the real-world setting $[4,5]$. A 'lateral switch' from fingolimod to another DMT may be necessary due to different reasons including lack of efficacy, family planning or safety issues. As guidelines do not make recommendation on this matter and clear data regarding treatment sequencing are missing, we consider it of utmost importance to give a compact overview of current data to help physicians in their daily routine when advising patients on fingolimod regarding a necessary 'lateral switch'.

Two general considerations have to be taken into account regarding efficacy when switching from one highly active DMT to another. First, the transition period between cessation of the existing DMT and initiation of a new agent must balance the efficacy, safety and the risk of recrudescence of disease activity or rebound. For instance, many real-world studies have reported disease reactivation after the discontinuation of fingolimod [6]. A second point for consideration is whether a previous drug could nullify or attenuate the mode of action of a subsequent therapy. Here, the question arises whether immune cell-depletion may be less effective if initiated immediately after the use of fingolimod as both the sequestration-dependent effects and those independent of sequestration are overlapping.

Beyond efficacy concerns, real-world data are also required to assess the potential impact of switching from one DMT to another on patient safety. As most active DMTs have lasting or even irreversible effects on patients' immune systems [7, 8], the cumulative impact of subsequent monotherapies with different mechanisms of action makes it difficult to predict the clinical consequences of highly active DMTs administered in succession.

Given the increasing complexity of treatment options and the fact that clear guidance to manage the switch from the second-line sequestering treatment agent fingolimod to other compounds is lacking, we here focus on the role of fingolimod pre-treatment on the efficacy and safety profile of the subsequent highly active agents used in RMS. We further comment on management considerations, such as baseline lymphocyte count and washout duration, to assist and optimize the decision-making process in clinical practice. Real-world and observational studies are herein reviewed to understand outcomes among patients who are potentially under-represented in controlled trials.

\section{Methods}

We conducted a MEDLINE search to identify relevant articles published between January 1, 2011 and August 31, 2021. The Medical Subject Headings (MeSH) terms applied were 'fingolimod' and 'switch' or 'escalation' or 'sequence' or 'natalizumab' or 'cladribine' or 'alemtuzumab' or 'ocrelizumab' or 'ofatumumab'. Additionally, we decided to include eligible studies sourced from international conferences (the Annual Meeting-American Academy of Neurology (AAN) and the European/Americas committee for treatment and research in multiple sclerosis (ECTRIMS/ ACTRIMS)), personal communications with the authors, and consultation of national and international registries for clinical trials [United States National Library of Medicine (NLM); clinicaltrials.gov; European Union Drug Regulating Authorities Clinical Trials Database (EudraCT)].

Articles that were included met the following criteria: (i) adult patients with active RMS according to 2010 or 2017 revised McDonald criteria [9, 10]; (ii) previous treatment with fingolimod according to national and international guidelines as well as the summary of product characteristic (SmPC); (iii) a switch to natalizumab, cladribine, alemtuzumab, ocrelizumab and ofatumumab.

Our literature search returned 1,059 records in total. Among these studies, 17 records met our inclusion criteria (for details on the search strategy see Fig. 1).

\section{Effectiveness and safety in patients switching from fingolimod to other DMT approved for active RMS}

Efficacy of fingolimod has been proven in various clinical trials [11-13]. However, in the real-world setting, up to 50\% of patients show ongoing disease activity within the first year of treatment $[14,15]$ and probably require a switch to another DMT. Furthermore, reasons like safety issues or family planning might make it necessary to switch DMT. Vollmer et al. did a 36-month follow-up on 535 fingolimod patients and observed that $21 \%$ of all patients discontinued fingolimod due to intolerance, $\sim 10 \%$ due to other reasons which might likely include family planning among others [16]. Wicks et al. performed an online survey in an online patient community [17]. 62 patients currently on fingolimod and 32 patient which discontinued fingolimod participated. $46.9 \%$ of the latter discontinued fingolimod treatment due to side effect, $25 \%$ due to of lack of effectiveness, $6.3 \%$ due to the advice of their doctor and $15.5 \%$ due to other reasons including family planning. 
Fig. 1 PRISMA flow diagram of the reviewed literature

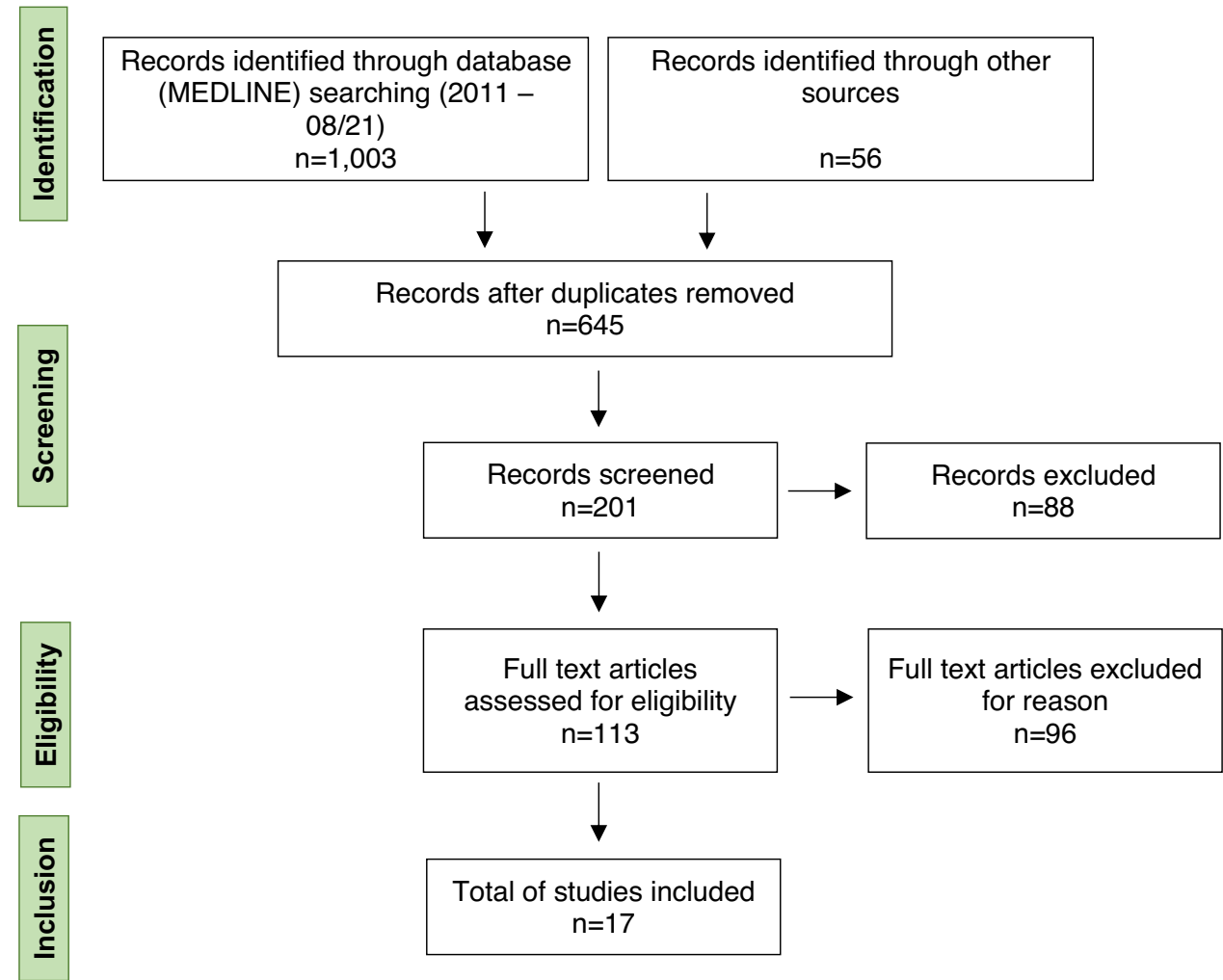

To ensure that disease activity was not predominantly driven by rebound following cessation of the last previous immunotherapy, re-baselining to month six was performed. Following fingolimod pre-treatment, patients experienced mainly paraclinical disease activity after the treatment switch; however, disease stability occurred for most patients after having passed month six. While early re-occurrence of disease activity during the washout period was confirmed by several case reports during the switch to cladribine [24, 25], the absence of disease reactivation after the initiation of treatment contrasted the results of Pfeuffer et al. [22]. Regarding safety considerations, both fingolimod and cladribine likely exert their clinical efficacy by depleting peripheral immune cells. However, fingolimod pre-treatment was neither a risk factor for the development of severe lymphopenia nor for the occurrence of herpes infection upon cladribine initiation [22].

\section{Switch to alemtuzumab}

Alemtuzumab was shown to be highly efficacious in controlling disease activity among both treatment-naïve patients (CARE-MS I) and those who had a poor response to a firstline DMT (CARE-MS II) [26, 27]. Patients enrolled in the CARE-MS II trial had been previously treated mainly with beta-interferon or glatiramer acetate; none of them received fingolimod [26]. 


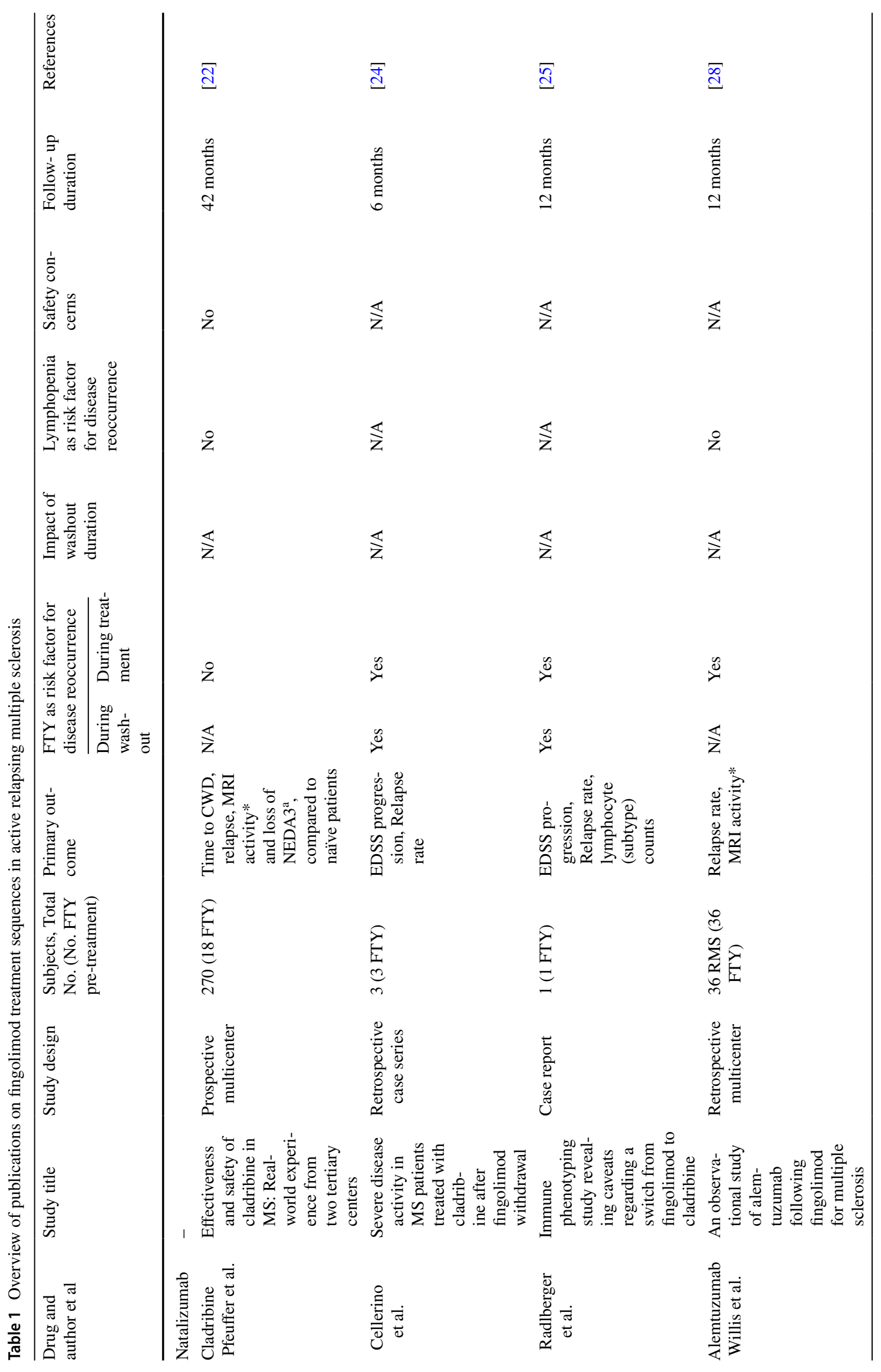




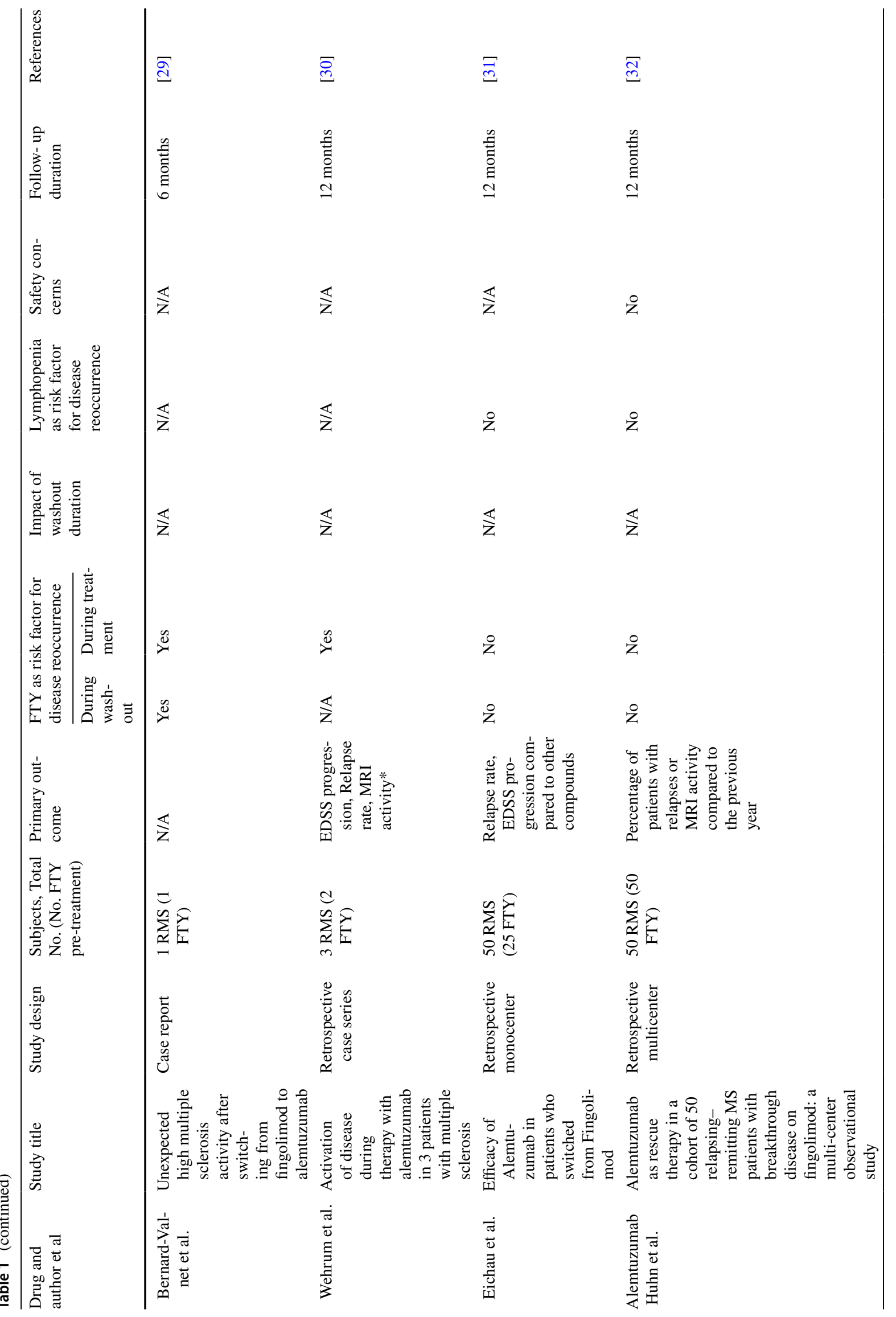




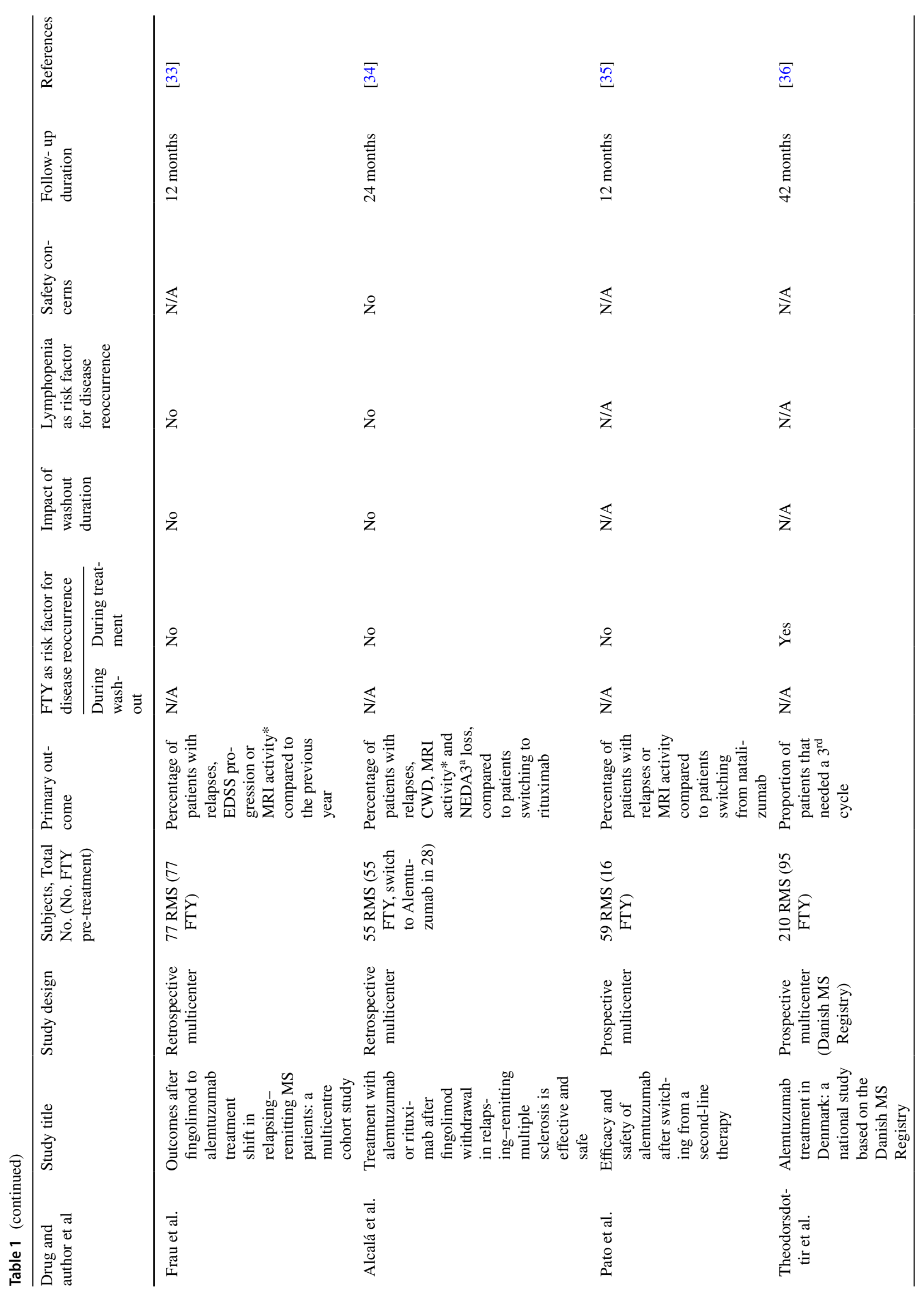




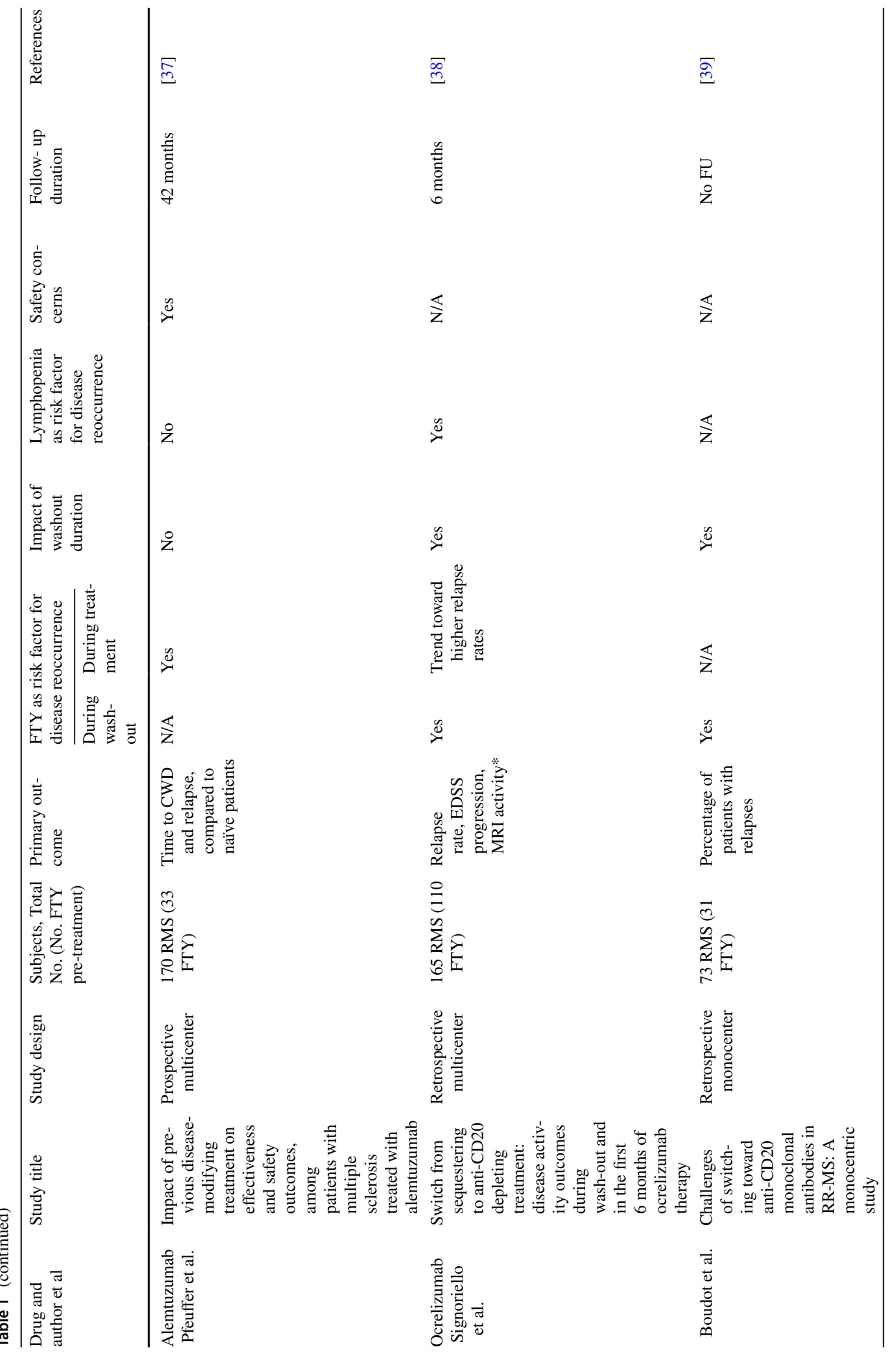




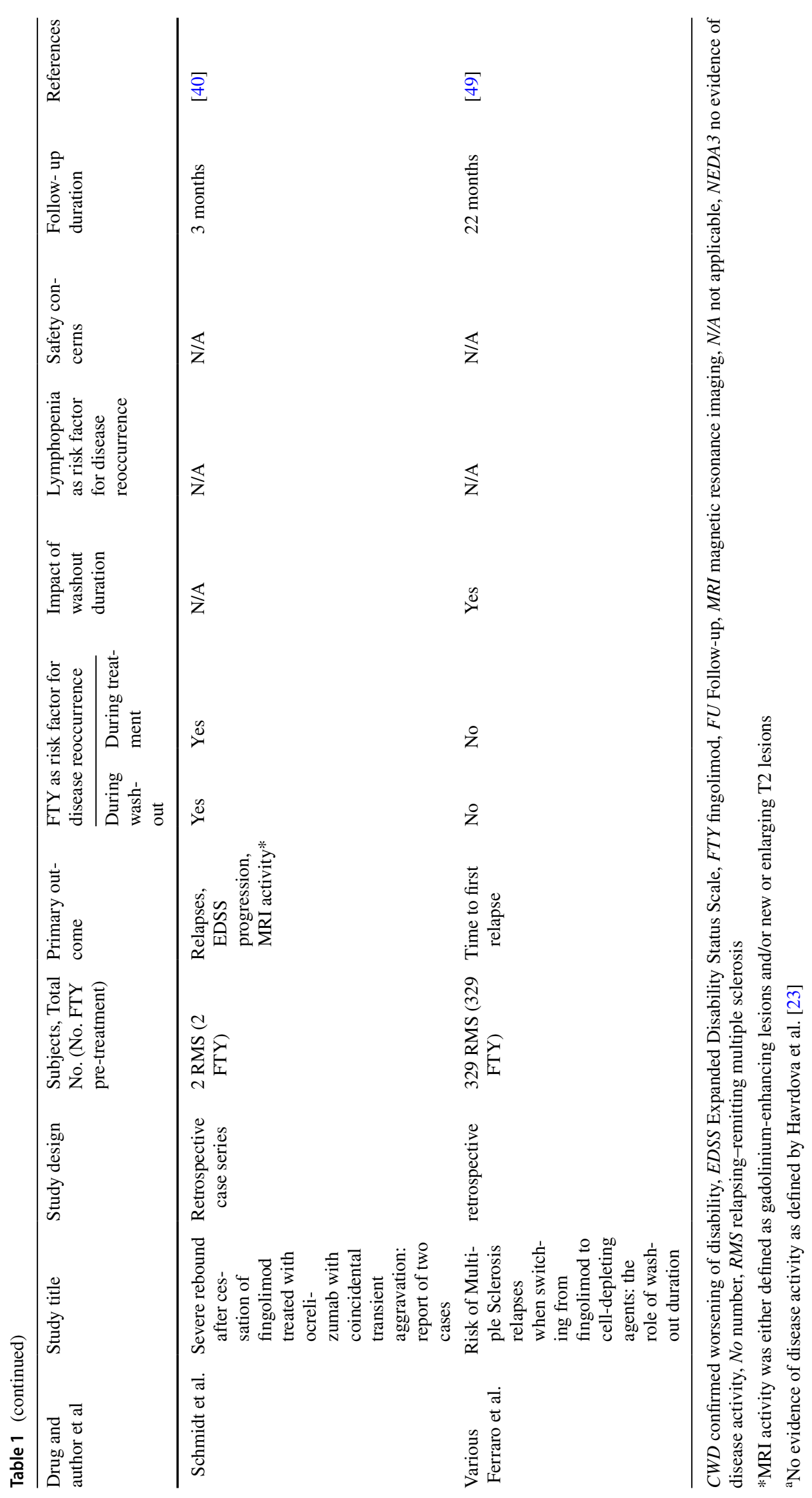


In the real-world setting, a British observational study by Willis et al. first raised concerns that the escalation to alemtuzumab might not achieve good control of disease activity [28]. The authors showed that 9 out of 36 patients experienced significant disease activity within the first twelve months after switching from fingolimod to alemtuzumab. While the mean relapse rate significantly increased during the first year of treatment, the second dose of alemtuzumab subsequently reduced the rate below pre-treatment values. In line with this, several case reports $[29,30]$ described significant and unexpected disease activity following switching from fingolimod, mainly around month six after alemtuzumab induction $[29,30]$. Subsequently, several studies were conducted to assess the efficacy of alemtuzumab in patients previously treated with fingolimod, with conflicting results. While four retrospective analyses [31-34] and one small prospective study [35] demonstrated good effectiveness of alemtuzumab following fingolimod, a Danish prospective registry study showed that patients previously exposed to fingolimod are more likely to require a third course of alemtuzumab due to ongoing disease activity compared to other compounds [36].

Notably, none of the aforementioned studies performed comparisons with previously naïve or platform-treated patients. In this context, we have recently published a large, prospective cohort study demonstrating that patients previously treated with fingolimod had a worse response to alemtuzumab compared with other treatment groups (including naïve patients and those who had received injectables), as they experienced a less marked reduction in relapse rate and higher hazard ratios for worsening disability [37]. Additionally, we observed an 'altered relapse pattern', as patients previously exposed to fingolimod were more likely to develop spinal relapses, contributing to their increased hazard radio for worsening disability.

Regarding the safety profile, and considering the contrasting results from three retrospective analyses mentioned above [32-34], we found that patients who had received fingolimod were prone to developing secondary autoimmunity, with no specific patterns of autoimmunity between treatment groups [37].

\section{Switch to anti-CD20 antibodies}

Regarding B cell depletion by anti-CD20 antibodies, an observational multicentre study recently assessed disease activity in 165 individuals treated with ocrelizumab following discontinuation of fingolimod or natalizumab [38]. The authors demonstrated that previous treatment with fingolimod increased relapses during washout and also noted a tendency toward increased relapse occurrence during the first 6 months of ocrelizumab therapy compared to patients switching from natalizumab. A similar trend of increased relapse rate during washout when switching from fingolimod to a B cell-depleting therapy compared to other compounds was further reported by Boudot et al. [39]. In this retrospective monocentre study, 20 patients $(27.4 \%$ of the total patients included) with different previous treatments experienced relapses during the washout period, with a probability of relapse post-fingolimod of 35\% 1 month after withdrawal.

In support of this, Schmidt et al. reported two cases of breakthrough disease during treatment with fingolimod who then experienced a severe rebound after fingolimod withdrawal with further significant clinical deterioration after ocrelizumab initiation [40].

It should be noted that a study has neither reported a favorable outcome when switching from fingolimod to ocrelizumab nor studied switching to ofatumumab-the safety profile of these treatment sequences is yet to be analyzed. Results from controlled clinical trials of ocrelizumab and ofatumumab are also unhelpful, as only 5 out of 825 patients in OPERA I and II [41] and 23 out of 946 patients in ASCLEPIOS I and II [42] were pre-exposed to fingolimod.

\section{Possible mechanisms of compromised efficacy and resulting management considerations}

Since a substantial proportion of lymphocyte subsets is sequestered in secondary lymphoid tissues by fingolimod, ongoing lymphopenia by sphingosine-1 phosphate receptor blockade probably results in reduced accessibility of cells for depletion and might therefore be a potential risk factor for suboptimal treatment response. Hu et al. showed that lymphocyte depletion by alemtuzumab was markedly less profound in lymphoid organs than in peripheral blood using a transgenic mouse model expressing human CD52 [43]. Regarding anti-CD20-agents experimental models have shown that there is likely a more direct access to lymph nodes with subcutaneous administration than with intravenous infusion $[44,45]$.

In total, seven of the included studies conducted a subgroup analysis regarding the absolute baseline lymphocyte count of patients with or without evidence of persistent clinical or MRI disease activity during subsequent treatment [22, 28, 32-34, 37, 38]. Indeed, Signoriello et al. showed that lymphocyte counts at baseline influence early treatment effectiveness of ocrelizumab [38]. However, in all other studies, lymphocyte counts at baseline did not differ significantly [22, 28, 32-34, 37]. Accordingly, in most of the studies indicating reduced effectiveness of either alemtuzumab $[28,29,37]$ or ocrelizumab $[38,40]$, lymphocyte counts were already at the lower limit of normal at the beginning of treatment. However, the total blood lymphocyte count does not depict differences in lymphocyte subsets and their respective tissue distribution. Yet, none of the included studies performed detailed immunophenotyping to address this 
issue. Only one case report described that re-emerging disease activity after cladribine was associated with accelerated repopulation of naïve $\mathrm{B}$ cells and lower pre-treatment $\mathrm{T}$ cell and memory B cell levels [25]. Similarly, recurrent disease activity under alemtuzumab treatment was associated with high B cell counts in three other patients [30].

Another widely debated aspect is the impact of the washout period on disease reactivation upon fingolimod discontinuation [46]. On the basis of a reversible immune reconstitution for fingolimod [47], and the risk of disease reactivation when washout is prolonged [48], a short period between two treatments could be considered to minimize rebound activity. Indeed, shortening the time period when switching to anti-CD20 therapies after fingolimod reduced relapses between the cessation and initiation of the new treatment $[38,39]$. Of note, this relationship was not investigated for other treatment switches.

On the other hand, and in line with aspects presented above, a longer washout period could lead to recovery from lymphopenia before initiation of cell-depleting agents, which may limit the risk of therapies being ineffective. Interestingly, a recent study by Ferraro et al. [49] in this journal showed that extending the washout period prior to commencing cell-depleting agents does not positively affect outcome parameters in the long term. In this study, the risk of relapse increased with the washout duration when switching from fingolimod to a lymphocyte-depleting agent (including cladribine, alemtuzumab, rituximab and ocrelizumab) during the 22 months of follow-up. In contrast, disease activity during alemtuzumab therapy did not correlate with the washout interval in other studies [33, 34, 37].

\section{Discussion}

Since prospective, randomized, controlled clinical trials are not available, this review summarized current real-world evidence regarding the best treatment switch strategy following fingolimod.

This current review highlights the dilemma that patients on fingolimod in need of a switch toward another DMT have few therapeutic options. It was shown that patients switching from fingolimod to ocrelizumab were more likely to experience suboptimal disease control and worsening of disability throughout the washout period and during a follow-up period of 6 month [38-40]. Use of alemtuzumab as successor to fingolimod was repeatedly debated [28-36]. However, a large prospective cohort study confirmed impaired effectiveness and safety of this therapeutic switch [37]. In contrast, cladribine remained effective and safe following fingolimod treatment [22], but-although no study yet examined the cumulative risk of adverse skin events-cladribine predisposes to dermatological side effects, including skin tumors
[50, 51], and thus concerns remain. Finally, natalizumab may remain an option but there is no real-world evidence yet that this switch is beneficial. Regarding feasibility and safety issues here, it should be considered that natalizumab carries a risk of progressive multifocal leukoencephalopathy (PML). Among fingolimod patients included in recent studies, up to $25 \%$ previously received and stopped natalizumab due to John Cunningham Virus (JCV) seropositivity and subsequent safety concerns $[52,53]$. Cases of PML occurred in patients receiving fingolimod in the post-marketing setting who had neither been previously treated with natalizumab nor were taking immunosuppressive or immunomodulatory medications concomitantly [54]. It is currently unknown whether or how the sequencing of these therapies might impact the overall PML risk for each patient. Data examining the outcome after a switch form fingolimod to another DMT in regards to the reason for the switch either being treatment inefficiency or safety concerns and personal matters such as family planning are missing. A differentiation between these patient groups appears crucial to the further understanding of an individualized treatment strategy.

Interestingly, one study analyzed the effect of re-dosing on disease activity [28] and demonstrated a reduced risk of relapse after the second dose of alemtuzumab. One explanation may be that after a period of time, sequestrated lymphocytes eventually become available for targeting. However, the contribution of lymphocyte sequestration by fingolimod to suboptimal disease control following cell-depleting treatment remains questionable and our literature search suggests this view is too simplistic. Further, no correlation between baseline $\mathrm{CD} 19^{+} \mathrm{B}$ cell levels and fingolimod pre-treatment in relapsing or stable patients were found upon ocrelizumab initiation [38].

However, it is likely that the cellular immune status (as performed in routine clinical practice) is not sufficient to determine the underlying mechanisms of disease re-occurrence. For example, in the past, a more detailed assessment of $\mathrm{B}$ cells in the periphery including $\mathrm{CD} 19^{+} \mathrm{CD} 27^{+}$memory $\mathrm{B}$ cells was shown to be more reliable in prediction of clinical activity [55]. In this context, potential similar memory $\mathrm{B}$ cell depletion mechanisms were further described with cladribine and alemtuzumab [56]. Moreover, previous data indicate that fingolimod also exerts several effects on B cells, including induction of a regulatory phenotype [57], reduction in memory B cells [58] and increased production of anti-inflammatory cytokines [59]. Therefore, during disease activity, it may be plausible that the removal of regulatory $\mathrm{B}$ cells from the peripheral immune system by the subsequent therapy contributes to relapses at the beginning of treatment. Conversely, pre-treatment with natalizumab was shown to not only increase peripheral $\mathrm{B}$ cell numbers but also induce a more pro-inflammatory phenotype of B cells [60]; patients undergoing the switch from natalizumab to ocrelizumab 
experienced sufficient disease control [38]. Other qualitative changes in the immune network that may influence disease activity include transcriptomic changes of $\mathrm{CD}^{+} \mathrm{T}$ cells [61], the modulation of $\mathrm{T}$ helper cell phenotype balances and the increase in regulatory $\mathrm{T}$ cell abundance [62]. We speculate that these effects interfere with cell depletion and immune reconstitution in an unfavorable manner, resulting in increased risk for progression of disability [24, 25, 28-30, 36-40] and, perhaps in part, the development of secondary autoimmunity [37].

\section{Conclusion and outlook}

There are numerous conclusions to be drawn from this review. First, the optimal successive treatment for patients with the need of a therapeutic switch while receiving fingolimod still needs to be defined. Evidence for natalizumab is lacking and, as it carries the risk of PML development, it may not be a longer-term alternative. Alemtuzumab also seems to represent a suboptimal option. Further studies evaluating whether ocrelizumab remains impaired in its effectiveness beyond re-treatment in month six and whether cladribine post-fingolimod is associated with a poor safety profile are necessary. Future studies should furthermore distinguish between the different reasons for treatment discontinuation. Efficacy after switching from fingolimod to another DMT may differ depending on the reason being disease activity or safety issues or family planning while the disease is basically stable.

Second, shortening of the washout period when switching from fingolimod may reduce relapse occurrence between cessation and initiation of the new treatment; however, regarding longer-term efficacy, data are contradictory. Therefore, further studies regarding the effect of blood lymphocyte subsets and their respective tissue distribution on the efficacy of the follow-up treatment after fingolimod are needed.

Finally, prescribing DMTs in RMS depends on a thorough risk-benefit analysis, which is inconclusive if the patient's characteristics are not reflective of clinical trial populations. Therefore, the question is raised whether patients with RMS previously treated with DMTs, other than what are considered platform agents, should be enrolled in clinical trials more regularly. Synthetic comparator arms for control groups could help to maintain feasibility here. Furthermore, it appears that DMTs more likely to require an 'ongoing evaluation' in the real-world setting, as new DMTs are continuously being introduced and consequently concerns regarding treatment switches cannot be clarified by extension studies alone. Here, implementation of international registries would add value to the existing evidence and improve data quality as the discussed data mainly originate from single center studies with smaller patient numbers. Such approaches would also be more likely to result in the recognition of safe succession of therapies. To date, data regarding 'safe successions' in a way represent negative reporting and it follows that they despite the best of intentions have been published less frequently.

In light of missing national and international guideline recommendations and taking all available real-world data into account, some centers prefer the following pathway: closely monitored washout period including immunmonitoring, lymphocyte counts above $800 / \mu$ l, if possible above $1000 / \mu l$ at initiation of new DMT, switch to B cell-depleting DMT after washout period.

As the treatment landscape for RMS evolves quickly, the question of recommendations on 'lateral switches' on patients under highly effective DMTs will become particularly important during the future. Therefore, high-quality data on efficacy and safety of 'lateral switches' and a joint concept how to collect these kinds of data internationally to obtain large patient numbers are needed.

Author contributions Conceptualization: MK, SP, SGM and H-PH. Literature search: MK, LR, SP; analysis and interpretation of data: MK, LR, SP, SGM and H-PH; data visualization and figures: LR; writingoriginal draft preparation: MK and LR; writing-review and editing: SGM and H-PH; critical revision of manuscript for intellectual content: SGM and H-PH; supervision: SGM and H-PH. All authors qualify for authorship according to current guidelines of Journal of Neurology. All authors have read and agreed to the submitted version of the manuscript. The current manuscript is not submitted to or under revision at another journal. All authors have read and agreed to the conditions noted on the Authorship Agreement Form.

Funding Open Access funding enabled and organized by Projekt DEAL. The authors received no financial support for research, authorship or publication of this article.

Availability of data and material As stated in the method section, data were obtained from MEDLINE, international conferences, personal communications with the authors, and consultation of national and international registries for clinical trials.

Code availability Not applicable.

\section{Declarations}

Conflicts of interest Melanie Korsen: received travel reimbursements from Merck Serono and Biogen; research support from Merck Serono and Novartis. Steffen Pfeuffer: received travel grants from Sanofi Genzyme and Merck Serono, lecturing honoraria from Sanofi Genzyme, Mylan Healthcare, and Biogen, and research support from Diamed, Merck Serono, and the German Multiple Sclerosis Society NorthrhineWestphalia. Leoni Rolfes: received travel reimbursements from Merck Serono and Sanofi Genzyme, research support from Diamed, Merck Serono and Novartis. Her research is funded by the Interdisciplinary Center for Clinical Studies (IZKF) Muenster. Sven G. Meuth: received honoraria for lecturing and travel expenses for attending meetings from Almirall, Amicus Therapeutics Germany, Bayer Health Care, Biogen, Celgene, Diamed, Genzyme, MedDay Pharmaceuticals, Merck Se- 
rono, Novartis, Novo Nordisk, ONO Pharma, Roche, Sanofi-Aventis, Chugai Pharma, QuintilesIMS, and Teva. His research is funded by the German Ministry for Education and Research (BMBF), Deutsche Forschungsgemeinschaft (DFG), Else Kröner Fresenius Foundation, German Academic Exchange Service, Hertie Foundation, Interdisciplinary Center for Clinical Studies (IZKF) Muenster, German Foundation Neurology, and by Almirall, Amicus Therapeutics Germany, Biogen, Diamed, Fresenius Medical Care, Genzyme, Merck Serono, Novartis, ONO Pharma, Roche, and Teva. Hans-Peter Hartung: received fees for consulting and serving on steering and data monitoring committees from Bayer Healthcare, Biogen, CSL Behring, Celgene Receptos, GeNeuro, MedDay, MedImmune, Merck, Novartis, Octapharma, Roche, Teva, TG Therapeutics, VielaBio Horizon Therapeutics.

The submitting author hereby declares that he takes responsibility for conduction and analysis of the data and that he had full access to all study data. The submitting author furthermore declares that there are no competing interests concerning these data and that the authors have all rights to publish the data. The submitted manuscript does not contain data that have been published in any other journal. The authors have no related articles under submission.

Open Access This article is licensed under a Creative Commons Attribution 4.0 International License, which permits use, sharing, adaptation, distribution and reproduction in any medium or format, as long as you give appropriate credit to the original author(s) and the source, provide a link to the Creative Commons licence, and indicate if changes were made. The images or other third party material in this article are included in the article's Creative Commons licence, unless indicated otherwise in a credit line to the material. If material is not included in the article's Creative Commons licence and your intended use is not permitted by statutory regulation or exceeds the permitted use, you will need to obtain permission directly from the copyright holder. To view a copy of this licence, visit http://creativecommons.org/licenses/by/4.0/.

\section{References}

1. McGinley MP, Goldschmidt CH, Rae-Grant AD (2021) Diagnosis and treatment of multiple sclerosis: a review. JAMA 325(8):765-779

2. Lorscheider $\mathbf{J}$ et al (2018) Comparative analysis of natalizumab versus fingolimod as second-line treatment in relapsing-remitting multiple sclerosis. Mult Scler 24(6):777-785

3. Baroncini D et al (2016) Natalizumab versus fingolimod in patients with relapsing-remitting multiple sclerosis non-responding to first-line injectable therapies. Mult Scler 22(10):1315-1326

4. Sormani MP, Laroni A (2017) Approved drugs for multiple sclerosis: the challenge of choice. Lancet Neurol 16(4):252-253

5. Hartung HP, Meuth SG, Thompson AJ (2021) Paradigm shifts: early initiation of high-efficacy disease-modifying treatment in multiple sclerosis. Mult Scler 27(10):1473-1476

6. Hatcher SE et al (2016) Rebound syndrome in patients with multiple sclerosis after cessation of fingolimod treatment. JAMA Neurol 73(7):790-794

7. Ruck $\mathrm{T}$ et al (2015) Alemtuzumab in multiple sclerosis: mechanism of action and beyond. Int J Mol Sci 16(7):16414-16439

8. Lunemann JD et al (2020) Immune reconstitution therapies: concepts for durable remission in multiple sclerosis. Nat Rev Neurol 16(1):56-62

9. Polman $\mathrm{CH}$ et al (2011) Diagnostic criteria for multiple sclerosis: 2010 revisions to the McDonald criteria. Ann Neurol 69(2):292-302

10. Thompson AJ et al (2018) Diagnosis of multiple sclerosis: 2017 revisions of the McDonald criteria. Lancet Neurol 17(2):162-173
11. Kappos L et al (2010) A placebo-controlled trial of oral fingolimod in relapsing multiple sclerosis. N Engl J Med 362(5):387-401

12. Chitnis $\mathrm{T}$ et al (2018) Trial of fingolimod versus interferon beta-1a in pediatric multiple sclerosis. N Engl J Med 379(11):1017-1027

13. Cohen JA et al (2010) Oral fingolimod or intramuscular interferon for relapsing multiple sclerosis. N Engl J Med 362(5):402-415

14. Barbin L et al (2016) Comparative efficacy of fingolimod vs natalizumab: a French multicenter observational study. Neurology 86(8):771-778

15. Pfeuffer S et al (2019) Efficacy and safety of alemtuzumab versus fingolimod in RRMS after natalizumab cessation. J Neurol 266(1):165-173

16. Vollmer B et al (2019) Comparative discontinuation, effectiveness, and switching practices of dimethyl fumarate and fingolimod at 36-month follow-up. J Neurol Sci 407:116498

17. Wicks $\mathrm{P}$ et al (2016) The real-world patient experience of fingolimod and dimethyl fumarate for multiple sclerosis. BMC Res Notes 9(1):434

18. Polman $\mathrm{CH}$ et al (2006) A randomized, placebo-controlled trial of natalizumab for relapsing multiple sclerosis. N Engl J Med 354(9):899-910

19. Rudick RA et al (2006) Natalizumab plus interferon beta-1a for relapsing multiple sclerosis. N Engl J Med 354(9):911-923

20. Giovannoni $\mathrm{G}$ et al (2010) A placebo-controlled trial of oral cladribine for relapsing multiple sclerosis. N Engl J Med 362(5):416-426

21. Giovannoni $\mathrm{G}$ et al (2018) Safety and efficacy of cladribine tablets in patients with relapsing-remitting multiple sclerosis: results from the randomized extension trial of the CLARITY study. Mult Scler 24(12): 1594-1604

22. Pfeuffer S et al (2021) Effectiveness and safety of cladribine in MS: Real-world experience from two tertiary centres. Mult Scler 13524585211012227

23. Havrdova E et al (2018) No evidence of disease activity (NEDA) analysis by epochs in patients with relapsing multiple sclerosis treated with ocrelizumab vs interferon beta-1a. Mult Scler J Exp Transl Clin 4(1):2055217318760642

24. Cellerino M et al (2020) Severe disease activity in MS patients treated with cladribine after fingolimod withdrawal. J Neurol Sci 418:117156

25. Radlberger RF et al (2021) Immune phenotyping study revealing caveats regarding a switch from fingolimod to cladribine. Mult Scler Relat Disord 48:102727

26. Coles AJ et al (2017) Alemtuzumab CARE-MS II 5-year followup: efficacy and safety findings. Neurology 89(11):1117-1126

27. Havrdova E et al (2017) Alemtuzumab CARE-MS I 5-year followup: durable efficacy in the absence of continuous MS therapy. Neurology 89(11):1107-1116

28. Willis M et al (2017) An observational study of alemtuzumab following fingolimod for multiple sclerosis. Neurol Neuroimmunol Neuroinflamm 4(2):e320

29. Bernard-Valnet $R$ et al (2018) Unexpected high multiple sclerosis activity after switching from fingolimod to alemtuzumab. Mult Scler Relat Disord 25:216-218

30. Wehrum $\mathrm{T}$ et al (2018) Activation of disease during therapy with alemtuzumab in 3 patients with multiple sclerosis. Neurology 90(7):e601-e605

31. Eichau S (2017) Efficacy of Alemtuzumab in patients who switched from Fingolimod. ECTRIMS Online Library. 200817; P1162

32. Huhn K et al (2018) Alemtuzumab as rescue therapy in a cohort of 50 relapsing-remitting MS patients with breakthrough disease on fingolimod: a multi-center observational study. J Neurol 265(7):1521-1527 
33. Frau J et al (2019) Outcomes after fingolimod to alemtuzumab treatment shift in relapsing-remitting MS patients: a multicentre cohort study. J Neurol 266(10):2440-2446

34. Alcala $\mathrm{C}$ et al (2019) Treatment with alemtuzumab or rituximab after fingolimod withdrawal in relapsing-remitting multiple sclerosis is effective and safe. J Neurol 266(3):726-734

35. Pato A, SSDM, Costa E, López Real A, Álvarez Rodriguez E, González Quintanilla V, González Suárez I, Álvarez L, Lorenzo JR, Arias Gómez M, Lema Devesa C, Amigo Jorrín MC, Peña Martínez J (2018) Efficacy and safety of alemtuzumab after switching from a second line therapy. ECTRIMS Online Library. Pato A. 10/12/18; 229053; P1213

36. Theodorsdottir A, MM, Schreiber KI, Kant M, Rasmussen PV, Hansen V, Bech D, Schmidt MF, Frederiksen JL, Sellebjerg F, Illes Z (2019) Alemtuzumab treatment in Denmark: a national study based on the Danish MS Registry. ECTRIMS Online Library. Theodorsdottir A. 09/13/19; 278551; P1349

37. Pfeuffer $\mathrm{S}$ et al (2021) Impact of previous disease-modifying treatment on effectiveness and safety outcomes, among patients with multiple sclerosis treated with alemtuzumab. J Neurol Neurosurg Psychiatry 92:1007-1013

38. Signoriello E et al (2021) Switch from sequestering to anti-CD20 depleting treatment: disease activity outcomes during wash-out and in the first 6 months of ocrelizumab therapy. Mult Scler. https://doi.org/10.1177/13524585211005657

39. Boudot de la Motte M et al (2021) Challenges of switching towards anti-CD20 monoclonal antibodies in RR-MS: a monocentric study. Mult Scler Relat Disord 52:102981

40. Schmidt S, Schulten T (2019) Severe rebound after cessation of fingolimod treated with ocrelizumab with coincidental transient aggravation: report of two cases. Ther Adv Neurol Disord 12:1756286419846818

41. Hauser SL et al (2017) Ocrelizumab versus interferon beta-1a in relapsing multiple sclerosis. N Engl J Med 376(3):221-234

42. Hauser SL et al (2020) Ofatumumab versus teriflunomide in multiple sclerosis. N Engl J Med 383(6):546-557

43. Hu Y et al (2009) Investigation of the mechanism of action of alemtuzumab in a human CD52 transgenic mouse model. Immunology 128(2):260-270

44. Kahari L et al (2019) Transcytosis route mediates rapid delivery of intact antibodies to draining lymph nodes. J Clin Invest 129(8):3086-3102

45. Theil D et al (2019) Imaging mass cytometry and single-cell genomics reveal differential depletion and repletion of B-cell populations following ofatumumab treatment in cynomolgus monkeys. Front Immunol 10:1340

46. Gross RH, Corboy JR (2019) Monitoring, switching, and stopping multiple sclerosis disease-modifying therapies. Continuum (Minneap Minn) 25(3):715-735

47. Francis G et al (2014) Temporal profile of lymphocyte counts and relationship with infections with fingolimod therapy. Mult Scler 20(4):471-480
48. Berger B et al (2015) Severe disease reactivation in four patients with relapsing-remitting multiple sclerosis after fingolimod cessation. J Neuroimmunol 282:118-122

49. Ferraro D et al (2021) Risk of multiple sclerosis relapses when switching from fingolimod to cell-depleting agents: the role of washout duration. J Neurol. https://doi.org/10.1007/ s00415-021-10708-1

50. Rolfes L et al (2021) Skin reactions in patients with multiple sclerosis receiving cladribine treatment. Neurol Neuroimmunol Neuroinflamm 8(3):e990

51. Mateo-Casas M et al (2020) Severe skin reactions associated with cladribine in people with multiple sclerosis. Mult Scler Relat Disord 43:102140

52. Kalincik T et al (2017) Treatment effectiveness of alemtuzumab compared with natalizumab, fingolimod, and interferon beta in relapsing-remitting multiple sclerosis: a cohort study. Lancet Neurol 16(4):271-281

53. Comi $\mathrm{G}$ et al (2020) Effectiveness of fingolimod in real-world relapsing-remitting multiple sclerosis Italian patients: the GENIUS study. Neurol Sci 41(10):2843-2851

54. Novartis G (2016) prescribing information. Novartis Pharmaceuticals Corporation, East Hanover

55. Lebrun C et al (2018) Only follow-up of memory B cells helps monitor rituximab administration to patients with neuromyelitis optica spectrum disorders. Neurol Ther 7(2):373-383

56. Baker D et al (2017) Both cladribine and alemtuzumab may effect MS via B-cell depletion. Neurol Neuroimmunol Neuroinflamm 4(4):e360

57. Grutzke B et al (2015) Fingolimod treatment promotes regulatory phenotype and function of B cells. Ann Clin Transl Neurol 2(2):119-130

58. Kemmerer CL et al (2020) Differential effects of disease modifying drugs on peripheral blood B cell subsets: A cross sectional study in multiple sclerosis patients treated with interferon-beta, glatiramer acetate, dimethyl fumarate, fingolimod or natalizumab. PLoS ONE 15(7):e0235449

59. Blumenfeld S, Staun-Ram E, Miller A (2016) Fingolimod therapy modulates circulating B cell composition, increases B regulatory subsets and production of IL-10 and TGFbeta in patients with Multiple Sclerosis. J Autoimmun 70:40-51

60. Traub JW et al (2019) Natalizumab promotes activation and proinflammatory differentiation of peripheral B cells in multiple sclerosis patients. J Neuroinflammation 16(1):228

61. Friess $\mathbf{J}$ et al (2017) Fingolimod alters the transcriptome profile of circulating CD4+ cells in multiple sclerosis. Sci Rep 7:42087

62. Dominguez-Villar M et al (2019) Fingolimod modulates $\mathrm{T}$ cell phenotype and regulatory $\mathrm{T}$ cell plasticity in vivo. $\mathrm{J}$ Autoimmun 96:40-49 